Original research article

\title{
Pharmacotherapy as major risk factor of falls - analysis of 12 months experience in hospitals in South Bohemia
}

\author{
Josef Maly ${ }^{1 *}$, Martin Dosedel ${ }^{1}$, Jan Vosatka ${ }^{1}$, Katerina Mala-Ladova ${ }^{1}$, Ales Antonin Kubena ${ }^{1}$, \\ Iva Brabcova ${ }^{2}$, Hana Hajduchova ${ }^{2}$, Sylva Bartlova ${ }^{2}$, Valerie Tothova ${ }^{2}$, Jiri Vlcek ${ }^{1}$ \\ ${ }^{1}$ Charles University, Faculty of Pharmacy in Hradec Králové, Department of Social and Clinical Pharmacy, Hradec Králové, Czech Republic \\ ${ }^{2}$ University of South Bohemia in Ceske Budejovice, Faculty of Health and Social Sciences, Institute of Nursing, Midwifery and Emergency Care, \\ České Budějovice, Czech Republic
}

\begin{abstract}
This study aimed to analyze the effect of fall risk-increasing drugs (FRIDs) and drug-related factors relative to falls through clinical pharmacy service in hospitalized patients, focusing on the relevance of clinical pharmacist evaluation in the context of physician assessment.

A prospective study of inpatient falls was conducted in 2017 retrieving data from 4 hospitals in South Bohemia, Czech Republic. An online database was developed to collect patient and fall-related data, and fall evaluation records. Healthcare professionals classified the overall effect of drugs on falls using Likert scale. Univariate and multivariate correlations were performed with a significance level of $p<0.05$.

Out of the total 280 falls (mean age of patients 77.0 years), a mean of 2.8 diagnoses with fall-related risk, 8.8 drugs, and 4.1 FRIDs per fall were identified. Incidence of falls decreased quarterly $(p<0.001)$. Use of FRIDs were positively associated with increasing age $(p=0.007)$. Clinical pharmacists were more likely to identify pharmacotherapy as the relevant fall-related risk, compared to physicians evaluation $(p<0.001)$. An increasing total number of prescribed drugs as well as higher number of FRIDs increased the suspicion in both professionals in the context of drug-related causes of falls.
\end{abstract}

Keywords: Clinical pharmacy service; Drug therapy; Fall risk-increasing drugs; Falls; Hospital inpatients

Abbreviations: FRID, fall risk-increasing drug

\section{Introduction}

Falls have become a major burden in healthcare facilities worldwide and are perceived as a significant public health concern (Aryee et al., 2017; Bloch et al., 2013). Falls, defined as the events resulting in a person coming to rest inadvertently on the ground, floor, or other lower level, belong globally among the leading causes of accidental or unintentional injury deaths (World Health Organization, 2018). Hospitalized patients are at high risk of accidental falls, considering from 1.4 to 17.9 falls per 1,000 days of hospitalization, depending on the type of healthcare facility, as well as on population characteristics (Lovallo et al., 2010). Falls-related complications increase patient morbidity and mortality, including prolonged hospitalization, early institutionalization, and reduced quality of life. The increase in financial costs of healthcare should not be forgotten either (Aryee et al., 2017; Bloch et al., 2013).

Patient falls often have a multifactorial character influenced by internal and external risk factors (Jung et al., 2014).
The major internal risk factors include higher age, some chronic and acute diseases, muscle weakness, fall history, impaired mobility, or decreased cognitive functions. External factors usually comprise precarious environment, hazardous activities and the use of certain drugs (de Jong et al., 2013; Deandrea et al. 2010; Hajduchová et al, 2016). Medications are generally proved as a fall-related risk factor. According to currently published systematic reviews, the most common fall risk-increasing drugs (FRIDs) are different types of drugs influencing the central nervous system (CNS) with a potential to affect vigilance, coordination, and balance (Seppala et al., 2018a), as well as cardiovascular drugs, which may induce syncope after hypotension (especially orthostatic), along with arrhythmia (Deandrea et al, 2010; de Vries et al., 2018). Nevertheless, other types of drugs might also increase the risk of falls (e.g. laxatives, antiepileptics, non-steroidal anti-inflammatory drugs, analgesics, or proton pumps inhibitors) (Seppala et al., 2018b). There is clear evidence that the combination of two or more risk drugs increases the risk of fall (Zia et al., 2017).

\footnotetext{
* Author for correspondence: Josef Maly, Charles University, Faculty of Pharmacy in Hradec Králové, Department of Social and Clinical Pharmacy, Akademika Heyrovského 1203, 50005 Hradec Králové, Czech Republic; e-mail: malyj@faf.cuni.cz http://doi.org/10.32725/jab.2019.001

Submitted: 2018-08-27 • Accepted: 2019-01-09 • Prepublished online: 2019-01-17

J Appl Biomed 17/1: 53-60 • EISSN 1214-0287 • ISSN 1214-021X

(c) 2019 The Authors. Published by University of South Bohemia in České Budějovice, Faculty of Health and Social Sciences.

This is an open access article under the CC BY-NC-ND license.
} 
Besides the pharmacological profile of individual drugs, interactions with pathophysiological changes associated with individual diseases also must be taken into consideration. Therefore, drugs influencing CNS, causing disorders of homeostasis and circulation (e.g. glycaemia, orthostatic hypotension), affecting muscle tone, as well as sensory functions, belong to the higher risk groups, as mentioned above. Moreover, the patients' individual reaction after drug administration is sometimes significantly different, compared to other patients (e.g. selective serotonin re-uptake inhibitors). Understanding these relationships is an essential prerequisite for reducing the risk of drug-related falls (Glab et al., 2014; Lord et al., 2011).

The knowledge of all the circumstances, including FRIDs, with a potential to raise the patients' overall risk of falls, early recognition of those factors, assessing their severity and finally, suggesting appropriate preventive strategies are a serious challenge to current clinical practice (Milos et al., 2014). Multifaceted cooperation may be advantageous, i.e. individual healthcare professionals should consider the extent of all risk factors at the time of any patient fall event. Guidelines suggest an expert should be invited, capable of rational reviews on dispensed medications (Beasley and Patatanian, 2009; Caffiero et al., 2017; Cheema et al., 2018; World Health Organization, 2018). The pharmacists' contribution consists in a comprehensive evaluation of pharmacotherapy to minimize its risks. In particular, effective preventive risk minimization tools may include the substitution of risk drugs, or proposing discontinuation of their administration (Beasley and Patatanian, 2009; Cheema et al., 2018; Chen et al., 2014).

The aim of the study was to analyse the effects of pharmacotherapy and drug-related factors on falls through clinical pharmacy service in hospitalized patients, focusing on the relevance of clinical pharmacist evaluation in the context of physician assessment.

\section{Materials and methods}

\section{Study design}

A prospective study of inpatients with fall events was conducted at 16 hospital wards (6x internal, $5 \times$ nursing, $2 \times$ surgical, $1 \times$ psychiatrist, $1 \times$ pneumology, and $1 \times$ rehabilitation unit) of four hospitals in South Bohemia region in the Czech Republic (CR). Hospital wards were selected based on the retrospective analysis of the highest number of falls reported in the period of 2014-2015 (Hajduchová et al., 2016). The current study included all inpatients with falls that occurred from January to December 2017, also on condition that have completed the data in the online database, as described below. Hospitalized patients of age 18 years and older only were eligible for the study. The research protocol was reviewed with respect to the strict rules of data protection and approved by the Ethical Committee of the Faculty of Pharmacy in Hradec Kralove, Charles University.

\section{Data collection}

The data collection online instrument (PharmaPortal EU) was constructed for the study to collect numerous variables on patients with fall events. The database enabled to record all the fall-related information, the patients' medical records data, as well as to communicate between healthcare professionals. The database management was supervised by a company specialized in protecting and storing sensitive data.

All data for the database were recorded by trained nurses, the accuracy verified by the treating physician, and an- onymized by a standard methodology. No personal information was known to researchers or published. The database was tested on a pilot sample (November and December 2016) to ensure the functionality and reliability when used by healthcare professionals; the pilot test results were not included in the final analysis. The primary investigators randomly checked $10 \%$ of all cases to confirm the correctness of the recorded data.

Data were collected on demographic characteristics, as well as on medical history (e.g. diagnoses, patients' current state) and medication. The drugs used by the patients were characterized according to ATC code, dose, route of administration, time of therapy initiation (long-term use: $>7$ days; new users: exact number of days), and drugs administered in the 24 hours prior to the fall event (e.g. symptom-relief drugs). Results of physical, biochemical and hematological examinations (e.g. blood pressure, pulse, body mass index, hemoglobin, serum sodium, potassium and chloride, glycaemia in diabetic patients after a fall, or estimated glomerular filtration), whenever relevant to the falls, were also included. Similarly, regarding the circumstances of the fall, data were collected on the location and time of the fall, the activity performed in the time of the fall, type of injury, state of consciousness immediately after the fall, internal (e.g. impaired balance in patient's history), and external causes of the fall (e.g. slipping), environmental factors, whether the fall was assisted by healthcare professional, examination and care conducted after the fall, the impact of the fall on the hospitalization, as well as fall prevention measures before and after the fall. Moreover, risk factors relevant to the evaluation of potential causes of the fall were recorded, such as the history of falls in the past 12 months, use of assistive devices (e.g. crutches, glasses), active smoking during hospitalization, history of alcohol abuse, or alcohol abuse prior to the fall.

Instead of narrative description of the fall and diagnoses with fall-related risk, the cases were filled in a structured form. The list of diagnoses with fall-related risk was generated based on the literature (Lord et al., 2011).

\section{Analysis of fall risk-increasing drugs}

The data analysis was primarily focused on identifying drugs and drug-related factors (external and internal) that can increase the risk of falls. Both physicians and clinical pharmacists became aware of all available patients' information mentioned above and expressed the possible drug-related cause of a fall event. The overall effect of the patients' pharmacotherapy on a given fall was assessed using 4-item Likert scale (yes -1 point; rather yes -2 ; rather not -3 , no -4 ). Moreover, clinical pharmacists (auditor and controller) detected population FRIDs and individual FRIDs for each patient. The population FRIDs were determined either based on the literature or by the mechanism of action, or they were proved of adverse drug reactions predicting the risk of fall. Population FRID was identified as individual FRID when it was not possible to unambiguously eliminate the effect of its use on a given fall, based on the expression of clinical pharmacists. In addition, clinical pharmacists have generated recommendations aimed at minimizing the risk of individual and population FRIDs sent to the treating physician via the database (a form of telemedicine/ pharmacy).

\section{Statistical analysis}

Data were processed by Wolfram, Mathematica, version 11.2 (Wolfram Research Inc.). Methods of descriptive statistics were performed for the metric items as the mean \pm standard deviation (SD), median, and range, with a $95 \%$ confidence 
interval (CI) in some cases. Relationships and dependencies were calculated by $\chi^{2}$, Mann-Whitney test, Kruskal-Wallis, Conover test, Kendall tau $(t)$ correlation as appropriate. Pareto analysis for population FRIDs was applied using the ATC codes recursively up to the second level; at each level, the frequency had to achieve at least $60 \%$ of drugs to proceed the next Pareto analysis of the lower ATC level. Multivariate analysis, specifically the tree-based technique (Krnacova et al., 2012), was performed to analyse dependencies of the metric variables (differences in the physicians' and pharmacists' opinions) on a larger number of non-independent binary variables (presence of FRIDs). $P$-value $<0.05$ was considered as statistically significant.

\section{Results}

\section{Baseline characteristics}

A total of 280 falls were included in the final analysis. Falls occurred within 3 (72; 25.7\%), 5 (102; 36.4\%), or 7 (130; 46.4\%) days after the beginning of hospitalization. The mean day of hospitalization, when the fall occurred, was $19.8 \pm 31.74$ days (median 8). Most patients (242; 86.4\%) fell once during the hospitalization; 14 patients fell twice, 2 three times, and 1 four times, respectively. The number of bed-days was relatively constant per each quarter in 2017 (59,533 vs. 57,912 vs. 56,229 vs. 56,661 bed-days), however, the total number of falls decreased significantly (122 vs. 61 vs. 50 vs. 47 falls) ( $p<0.001)$.

There were 143 (51.1\%) falls in females. The mean age of patients with falls was $77.0 \pm 12.0$ years ( $\min 27$, $\max 95$ ), in females $79.3 \pm 11.1$ years, and $74.6 \pm 12.4$ years in males. At lower age, falls were significantly more frequent in males than in females $(p<0.001)$. Overall, patients with fall were treated by a mean of $8.8 \pm 4.2$ drugs (median 8 ; $\min 1$, $\max 24$ ). Females used significantly more drugs (mean $9.3 \pm 4.5$; median 9) than males (mean $8.2 \pm 3.7$ drugs; median 8$)(p=0.027)$.

A total of 775 fall risk-increasing diagnoses were identified, i.e. on average $2.8 \pm 1.4$ (median 3 ; $\min 0$, $\max 7$ ) per fall. The most common risk diagnoses (with at least $20.0 \%$ of falls) were arterial hypertension (194; 69.3\%), diabetes mellitus (93; 33.2\%), tachycardia (86; 30.7\%), and dementia including Alzheimer disease $(56 ; 20.0 \%)$. Other selected fall-related risk factors are summarized in Table 1.

\section{Analysis of pharmacotherapy in relation to falls}

Concerning the pharmacotherapy in relation to falls, patients used 1.134 population FRIDs, i.e. a mean of $4.1 \pm 2.4$ per fall (median 4; min 0, max 13). Most of population FRIDs (994; $87.7 \%$ ) were used chronically. Each drug of a patient was associated with 0.41 population FRIDs (CI 95\% = 0.36-0.46). The number of population FRIDs was higher in increasing age ( $t=0.113, p=0.007$ ), even if the total number of drugs used by the patients was not associated with their age $(p=0.075)$. No significant difference among gender in relation to the use of population FRIDs was detected $(p=0.323)$. Patients with previous fall experience did not use significantly less population FRIDs $(p=0.629)$. Clinical pharmacists evaluated $498(43.9 \%)$ population FRIDs as individually risky, i.e. that patients were taking $1.8 \pm 1.7$ individual FRIDs per fall (median 1 ; min 0 , $\max 9$ ) on average. No demographic characteristics were significantly associated with individual FRIDs.

Based on Pareto analysis, $60 \%$ of falls were associated with population FRIDs from " $N$ " (Nervous System) and "C" (Cardiovascular System) groups according to the first level of ATC clas-
Table 1. Fall-related risk factors $(N=280)$

\begin{tabular}{lr}
\hline & $N(\%)$ \\
\hline polypharmacy (4 and more drugs) & $246(87.9)$ \\
age over 65 years & $233(83.2)$ \\
two and more population fall risk-increasing drugs & $230(82.1)$ \\
$\begin{array}{l}\text { no other external factors were found indicating the cause } \\
\text { of the fall }\end{array}$ & $224(80.0)$ \\
$\begin{array}{l}\text { time when the fall occurred was most probably related to } \\
\text { the use of drug }\end{array}$ & $112(40.0)$ \\
$\begin{array}{l}\text { fall history in the past } 12 \text { months } \\
\text { assistive devices used by the patient (e.g. crutches) }\end{array}$ & $110(39.3)$ \\
history of alcohol abuse or alcohol abuse during the fall & $22(7.9)$ \\
active smoking during hospitalization & $21(7.5)$ \\
\hline
\end{tabular}

sification. The most commonly used drugs from those groups are demonstrated in Tables 2 and 3.

\section{Physician and clinical pharmacist opinions}

Clinical pharmacists were more likely to attribute the fall-related risk to the use of drugs, compared to the physicians' opinion ( $t=0.392, p<0.001)$, as shown in Table 4 , nevertheless, the concordance coefficient in rating was low $\left(\rho_{c}=0.354\right)$. The mean score of clinical pharmacists' and physicians' opinion was $2.4 \pm 0.8$ (median 2) and $3.0 \pm 0.8$ (median 3), respectively (Fig. 1). However, in 17 (6.1\%) falls physicians suggested higher importance of drug-related cause, compared to clinical pharmacists.

Based on physicians' opinion, in general, there were no groups of all prescribed drugs (the first level of ATC classification considered) identified as a predictive risk factor of fall. On the other hand, drugs from the group " $N$ " in the patient's history revealed a higher chance to cause falls according to the opinion of clinical pharmacists $(p=0.002)$. Moreover, if a drug from the group " $N$ " was not used by the patient, a drug from the group " $C$ " was then considered by clinical pharmacists as a predictive factor $(p=0.002)$.

Further, the population FRIDs according to ATC classification and the risk of fall are demonstrated in Fig. 2. The population FRIDs from the group " $N$ " reveal a higher chance to cause the fall, based on both physicians' and clinical pharmacists' opinion. The difference between their opinions was significant, in favor of the pharmacists $(p=0.026)$. Indeed, if there was no population FRIDs from the group " $N$ ", population FRIDs from " $C$ " were predicted as a fall-related risk factor by both groups of professionals $(p=0.012)$. If the patient was not treated by any population FRIDs, neither from " $N$ ", nor " $C$ " group, the difference between the physicians' and clinical pharmacists' opinions was completely negligible.

Generally, an increase in the number of drugs used by patients significantly raised the suspicion in both physicians and clinical pharmacists, in relation to the drug-related cause of falls. Physicians were significantly more aware of the possible effects both of the total drugs $(t=0.157, p=0.004)$ and of population FRIDs ( $t=0.179, p=0.002)$ on falls. Similar results were observed in clinical pharmacists' suspicions $(t=0.184$, $p<0.001$, and $t=0.224 ; p<0.001$, respectively). However, the difference between opinions of those involved health professionals was not statistically significant concerning the total number of drugs $(p=0.294)$, or population FRIDs $(p=0.132)$. 
Table 2. The most frequent population and individual fall risk-increasing drugs from the group " $N$ " according to the ATC classification $(N=280)$

\begin{tabular}{|c|c|c|c|}
\hline & $\begin{array}{c}\text { Drugs with } \\
\text { population risk } N(\%)\end{array}$ & $\begin{array}{l}\text { Drugs with individual } \\
\text { risk } N(\%)\end{array}$ & $I / P$ in $\%$ \\
\hline N05 - psycholeptics & $193(68.9)$ & $145(51.8)$ & $75.1 \%$ \\
\hline N05A - antipsychotics, neuroleptics & $132(47.1)$ & $94(33.6)$ & $71.2 \%$ \\
\hline N05BA - benzodiazepines & $43(15.4)$ & $35(12.5)$ & $81.4 \%$ \\
\hline N05BB01 - hydroxyzine & $2(0.7)$ & $2(0.7)$ & $100.0 \%$ \\
\hline N05C - hypnotics and sedatives & $16(5.7)$ & $14(5.0)$ & $87.5 \%$ \\
\hline N06 - psychoanaleptics & $109(38.9)$ & $40(14.3)$ & $36.7 \%$ \\
\hline N06AB - selective serotonin reuptake inhibitors & $66(23.6)$ & $14(5.0)$ & $21.1 \%$ \\
\hline N06AX05 - trazodone & $15(5.4)$ & $11(3.9)$ & $73.3 \%$ \\
\hline N06AX11 - mirtazapine & $8(2.9)$ & $5(1.8)$ & $62.5 \%$ \\
\hline N06AX14 - tianeptin & $4(1.4)$ & $4(1.4)$ & $100.0 \%$ \\
\hline N06AX16 - venlafaxine & $4(1.4)$ & $2(0.7)$ & $50.0 \%$ \\
\hline N06D - antidementia drugs & $11(3.9)$ & $4(1.4)$ & $36.4 \%$ \\
\hline N02 - analgetics & $52(18.6)$ & $40(14.3)$ & $76.9 \%$ \\
\hline N02AA-F - opioids & $15(5.4)$ & $15(5.4)$ & $100.0 \%$ \\
\hline N02AX - tramadol & $34(12.1)$ & $22(7.9)$ & $64.7 \%$ \\
\hline N02BE71 - acetaminophen, combination with psycholeptics & $3(1.1)$ & $3(1.1)$ & $100.0 \%$ \\
\hline N03 - antiepileptics & $48(17.4)$ & $37(13.2)$ & $77.1 \%$ \\
\hline N03AE01 - clonazepam & $9(3.2)$ & $8(2.9)$ & $88.9 \%$ \\
\hline N03AF01 - carbamazepine & $4(1.4)$ & $3(1.1)$ & $75.0 \%$ \\
\hline N03AX12, 16 - gabapentin, pregabalin & $25(8.9)$ & $18(6.4)$ & $72.0 \%$ \\
\hline N04 - anti-Parkinson drugs & $19(6.8)$ & $16(5.7)$ & $84.2 \%$ \\
\hline N07 - other nervous system drugs & $2(0.7)$ & $0(0)$ & $0 \%$ \\
\hline
\end{tabular}

Table 3. The most frequent population and individual fall risk-increasing drugs from the group " $C$ " according to the ATC classification $(N=280)$

\begin{tabular}{|c|c|c|c|}
\hline & $\begin{array}{c}\text { Drugs with } \\
\text { population risk } N(\%)\end{array}$ & $\begin{array}{l}\text { Drugs with individual } \\
\text { risk } N(\%)\end{array}$ & $I / P$ in $\%$ \\
\hline C03 - diuretics & $169(60.4)$ & $70(25.0)$ & $41.4 \%$ \\
\hline C03A, C03B, C03E - thiazides + thiazide-like diuretics & $14(5.0)$ & $5(1.8)$ & $35.7 \%$ \\
\hline C03C - furosemide & $97(34.6)$ & $45(16.1)$ & $46.4 \%$ \\
\hline C03D - spironolactone & $58(20.7)$ & $20(7.1)$ & $34.5 \%$ \\
\hline C07 - beta blocking agents & $146(52.1)$ & $39(13.9)$ & $26.7 \%$ \\
\hline C09 - agents acting on the renin-angiotensin system & $87(31.1)$ & $27(9.6)$ & $31.0 \%$ \\
\hline C08 - calcium channel blockers & $55(19.6)$ & $16(5.7)$ & $29.1 \%$ \\
\hline C01 - cardiac therapy & $53(18.9)$ & $14(5.0)$ & $26.4 \%$ \\
\hline C01BD - antiarrhythmic agents I., III. class & $23(8.2)$ & $4(1.4)$ & $17.4 \%$ \\
\hline C01AA05 - digoxin & $19(6.8)$ & $3(1.1)$ & $15.8 \%$ \\
\hline C01AD - organic nitrates & $10(3.6)$ & $7(2.5)$ & $70.0 \%$ \\
\hline C02 - antihypertensive agents & $16(5.7)$ & $4(1.4)$ & $25.0 \%$ \\
\hline C02CA06 - urapidil & $8(2.9)$ & $3(1.1)$ & $37.5 \%$ \\
\hline C02AC - imidazoline receptor agonists & $8(2.9)$ & $1(0.4)$ & $12.5 \%$ \\
\hline C10 - lipid modifying agents & $2(0.7$ & $1(0.4)$ & $50.0 \%$ \\
\hline
\end{tabular}

Table 4. Drugs as fall-related cause by view of physicians and clinical pharmacists $(N=280)$

\begin{tabular}{lcr}
\hline Drug-related cause of the fall & Physicians, $N(\%)$ & Clinical pharmacists, $N(\%)$ \\
\hline yes & $13(4.6)$ & $27(9.6)$ \\
rather yes & $51(18.2)$ & $129(46.1)$ \\
rather no & $140(50.0)$ & $87(31.1)$ \\
no & $73(26.1)$ & $17(6.1)$ \\
not applicable & $3(1.1)$ & $20(7.1)$ \\
\hline
\end{tabular}




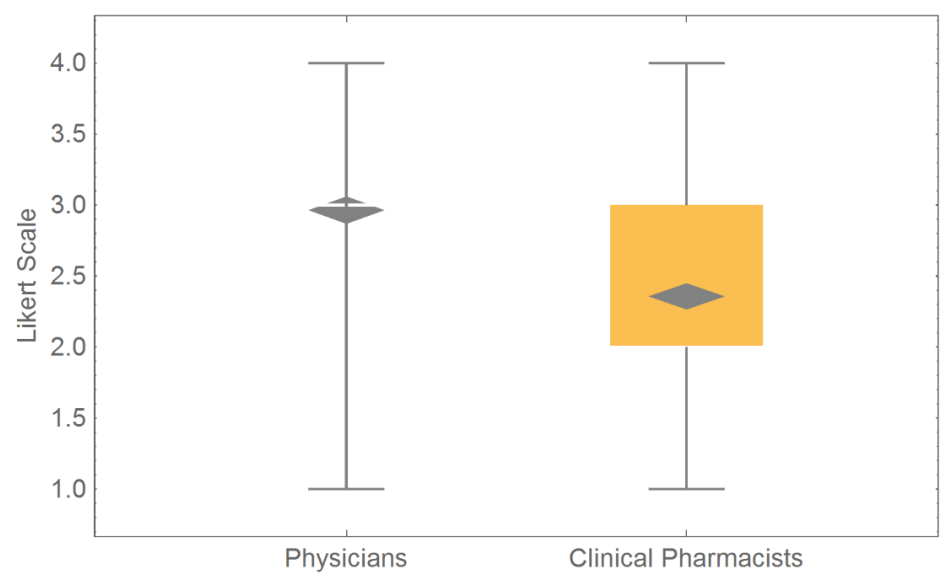

Fig. 1. Different opinions on drugs as fall-related causes

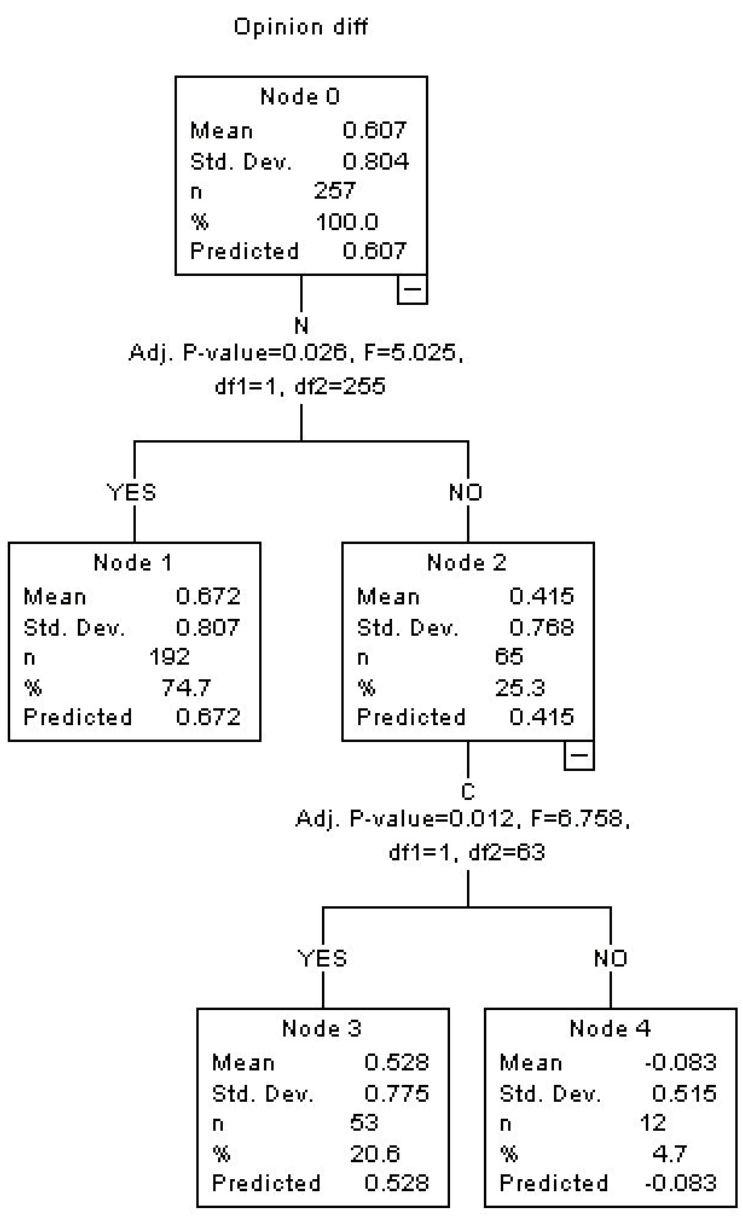

Fig. 2. Different physicians' and clinical pharmacists' opinions on drug-related cause of the fall by regression tree

\section{Discussion}

Our project reflects the multifactorial character of falls, and also the fact that multicomponent interventions are essential to manage this problem including drug-related causes. One of the preventive interventions is the comprehensive interdisciplinary collaboration which enables to better assess risks and benefits of pharmacotherapy, as well as to optimize it, while achieving the intended goals of each individual therapy (Hunderfund et al., 2011; Miake-Lye et al., 2013).

Data on falls have been collected for a long time in the Czech Republic. The Czech national database containing longitudinal data of falls characterized as extraordinary events was first launched at the Medical Faculty of Charles University, and subsequently at the Institute of Health Information and Statistics in the CR (Institute of Health Information and Statistics of the Czech Republic, 2018). Reports from this database comprised mostly sedative drugs such as benzodiazepines, opioids, hypnotics, and further, antihypertensive drugs and insulins, therefore, physicians and nurses may be insufficiently aware of other drugs that can lead to the falls.

It is important to know both the mechanisms of action of FRIDs and the patient's individual reactivity when the specific drugs are being assessed. In our cohort, there were FRIDs with different mechanisms concerning the occurrence of fall. In accordance with published literature (Lord et al., 2011), patients most frequently took drugs reducing mental alertness or inducing sedation, confusion, orthostatic hypotension $(\mathrm{OH})$, rhythm disturbances, eventually hypoglycemia. As it can be derived from Table 2, the prevalence of population FRIDs influencing CNS was high in our patients. However, benzodiazepines and strong opioids were surprisingly less represented among the sedative FRIDs (only $15.4 \%$ and $5.4 \%$, respectively), while Z-hypnotics and drugs used for neuropathic pain (gabapentin, pregabalin, carbamazepine) were more commonly identified. Our results suggest that physicians are likely to perceive some drugs as carrying higher risk, and after contacting the clinical pharmacist, they may gain information about other FRIDs. This signal is amplified by the fact that patients with a fall history did not use more population FRIDs than patients without falls. Moreover, for instance, drugs for neuropathic pain do not signalize the influence of the CNS, therefore healthcare professionals may not perceive them as 
being at risk at all. Similarly, the fall-related risk of antidepressants which have been increasingly used in the treatment of anxiety, depression, and in sleep disorders, such as trazodone or mirtazapine, can be underestimated. Mostly due to poor experience of their use in clinical practice and thus the low awareness of CNS attenuation (except for psychiatrists). The explanation of non-psychiatric indications can be more probable, as we included only one psychiatric ward in our study, as well as because such FRIDs were prescribed by other specialists. A further example of potentially underestimated risk can be perceived in the relatively high utilization of antipsychotics, posing two mechanisms of risk of fall. In addition to the CNS attenuation, they cause alpha1 blockade inducing hypotension including $\mathrm{OH}$. Among others, the rationality of prescriptions has to be taken into account as well (Mackin, 2008). Tramadol, often not identified by physicians and nurses among FRIDs, is a special case. It affects opioid receptors causing CNS attenuation, and further it can increase confusion in patients by inducing serotonin effect (Söderberg et al., 2013).

Besides the FRIDs influencing CNS, clinical pharmacists pointed out to FRIDs based on $\mathrm{OH}$ mechanism. Cardiovascular drugs were highlighted, especially angiotensin converting enzyme inhibitors (ACEI), which are quite often prescribed in clinical practice, just as well in our study. The risk occurs by influencing peripheral resistance, preload, and afterload. A lower risk of $\mathrm{OH}$ is associated with the use of alpha1-sympatholytics, which directly affect the vasodilatation ability of vessels, and increase sympathetic tone when changing body position. Tamsulosin intended for treatment of benign prostatic hyperplasia (therefore included in another ATC group) has been widely used FRID with this mechanism. Although, this sympatholytic agent is partially organ specific, it can induce $\mathrm{OH}$ in highly sensitive patients. To our knowledge, there is little awareness that this drug could affect circulation, due to its different indication. Cardiovascular drugs also comprise beta blockers inducing $\mathrm{OH}$ via lowering cardiac volume and reducing the effect of the sympathetic nervous system on cardiac functions. Diuretics also belong among common FRIDs, which decrease blood volume and therefore preload. Preload can also be reduced by vasodilation of the capacity vessels, as demonstrated by nitrates in our study. Overall, the above mentioned FRIDs have been used in combination with each other, as evidenced by high prevalence of arterial hypertension, also with other FRIDs potentially causing $\mathrm{OH}$, therefore the fall-related risk was even higher (IBM Micromedex, 2018; Lord et al., 2011).

In addition to other most frequent diagnoses, the proarrhythmic and hypoglycemic effects of pharmacotherapy can be associated with risk of falls. The first one is revealed in both antiarrhythmic agents (digoxin) and some other drugs, such as citalopram, antipsychotics, or theophylline. Hypoglycemia can develop mainly due to sulfonylurea-type antidiabetics, as well as insulin, even if the effect size can vary considerably.

Generally, not only FRIDs, but also other risk factors that may contribute to drug-related falls are advisable to be monitored. We have noticed that nurses were reporting less precisely the patients' status of hydration. Hydration is an important risk factor concerning falls, even if it is easy to be well-monitored, optimally in all hospitalized patients. Great emphasize should be given at least in patients taking $\mathrm{OH}$ inducing drugs, or with a history of hypotension. From our sample, signals from medication review highlighted loop diuretics (furosemide) in relation to dehydration. Therefore, it is necessary to ensure rational prescribing of furosemide, i.e. its prescription should always be considered, whether it is useful for the indi- vidual patient. Higher age even increases the fall-related risk, thus furosemide belongs among potentially inappropriate drugs in the elderly (Okada et al., 2017). Individual evaluations of population FRIDs should also include the drug onset, duration of the effect, and the length of drug administration, all of which depend on pharmacokinetic and pharmacodynamic properties. As examples, the so-called first-dose effect of ACEI, $\alpha$-sympatholytic drugs, or some sedative drugs could be mentioned (Walker and Whittlesea, 2012).

The benefits of a clinical pharmacist as an expert on drug management in various healthcare settings are evident (Cheema et al., 2018), however, there is no published, clear evidence about clinical pharmacy service in context of falls in the Czech Republic, and not even in abroad. Since drugs are a modifiable risk factor of falls, medication review should be incorporated in fall prevention programs (de Jong et al., 2013), and therefore it is important to strengthen pharmacist-conducted interventions in minimization of drug-related falls in both ambulatory (Bartlett et al., 2015; Mott et al., 2016) and inpatient settings in all risk groups (Browne et al., 2014; Marvin et al., 2017). Moreover, Marvin et al. showed that pharmacist involvement in medication review in fact resulted in a significant reduction in the number of FRIDs per patient (Marvin et al., 2017).

Underestimating the drug-related cause of falls is not appropriate (Ribeiro et al., 2018). On the other hand, it is necessary to recall the multifactorial nature of falls, which makes the assessment difficult. Compared to clinical pharmacists, the physicians' advantage could be seen in their being in daily contact with the patients, and the knowledge of the patients' current health condition. Therefore, pharmacists should also be present at the hospital ward in person, thus would not have to rely on indirect information (via interactive database). This fact may have played a role in cases when physicians expressed higher importance of some FRIDs than clinical pharmacists. However, physicians are generally less focused on the risks of pharmacotherapy in comparison with pharmacists. This can be related to the fact that pharmacists are more intensively educated in the whole spectrum of drugs. In addition, measuring the influence of most risk factors is very difficult, as well as multiple factors can interact with each other. It is therefore necessary that all three groups of healthcare professionals (physicians, pharmacists, and nurses) should cooperate in prevention and solution of falls, and the final interventions ought to be the result of their consensus.

Interestingly, the number of falls longitudinally decreased during the study, which was not random and probably caused by several factors. The project itself was intended to raise awareness of this issue, as well as stimulate discussion among healthcare professionals. Further, clinical pharmacists' interventions could positively affect physicians' behavior in terms of minimizing the risks of population FRIDs. The study therefore promotes safe medication practice too (Weddle et al., 2017). The follow-up analysis of patients without history of falls might show to what extent the interdisciplinary approach plays a role, and whether the prescribing habits concerning FRIDs have been actually changed.

The patients' individual responses to the pharmacotherapy can be identified as the main limit of the study, which could increase or reduce the drug-related risk of fall. Regarding data collection, it was not always possible to obtain all current data about the patients' conditions (e.g. hydration). We cannot forget the time-consuming data collection into a database, however, no significant errors were found during random controlling. The large amount of data allowed us to focus solely on the FRIDs and drug-related factors, therefore our results 
generated signals of drug interaction with the patients' various morbidity, which should be confirmed by future analysis. Apart from this, the knowledge and skills of healthcare professionals are only estimated based on available data and will be evaluated in an ongoing study. Cross-sectional study does not enable to observe risk factors (health condition, pharmacotherapy), which could play a role within the time period (e.g. before fall) as well as this type of study cannot identify rarely occurring phenomena.

\section{Conclusions}

Decreasing number of falls we experienced during the study period confirmed the importance of multidisciplinary collaboration between physicians, nurses, and pharmacists to minimize the risk of falls in healthcare facilities. This could enable a more accurate assessment of all the circumstances of falls, such as properties and risks of pharmacotherapy, and arranging the rational options how to eliminate these risks in the context of the individual patient. In the present study, higher age of the patients, increasing number of all prescribed drugs, increasing number of population FRIDs, and population FRIDs from ATC group affecting the nervous and cardiovascular system have been shown to be the significant predictors of drug-related falls. Compared to physicians, clinical pharmacists predicted more frequently the drug-related causes of the fall, especially antipsychotics, antidepressants, opioids, antiepileptics, and benzodiazepines being considered.

\section{Conflict of interests}

The authors declare no conflicts of interests to disclose.

\section{Acknowledgement}

Supported by the Ministry of Health of the Czech Republic Grant No. 16-33463A. All rights reserved.

\section{References}

Aryee E, James SL, Hunt GM, Ryder HF (2017). Identifying protective and risk factors for injurious falls in patients hospitalized for acute care: a retrospective case-control study. BMC Geriatr 17: 260. DOI: 10.1186/s12877-017-0627-9.

Bartlett D, Pang N, Massey C, Evans P (2015). Pharmacist consultations: simplifying daily drug regimens and providing education on fall risk for older adults. Consult Pharm 30(3): 141-152. DOI: 10.4140/TCP.n.2015.141.

Beasley B, Patatanian E (2009). Development and implementation of a pharmacy fall prevention program. Hosp Pharm 44(12): 1095-1102. DOI: 10.1310/hpj4412-1095.

Bloch F, Thibaud M, Tournoux-Facon $C$, Brèque $C$, Rigaud AS, Dugué B, Kemoun G (2013). Estimation of the risk factors for falls in the elderly: can meta-analysis provide a valid answer? Geriatr Gerontol Int 13(2): 250-263. DOI: 10.1111/j.14470594.2012.00965.x.

Browne C, Kingston C, Keane C (2014). Falls prevention focused medication review by a pharmacist in an acute hospital: implications for future practice. Int J Clin Pharm 36(5): 969-975. DOI: 10.1007/s11096-014-9980-3.

Caffiero N, Delate T, Ehizuelen MD, Vogel K (2017). Effectiveness of a clinical pharmacist medication therapy management program in discontinuation of drugs to avoid in the elderly. J Manag Care Spec Pharm 23(5): 525-531. DOI: 10.18553/jmcp.2017.23.5.525.
Cheema E, Alhomoud FK, Kinsara ASA, Alsiddik J, Barnawi MH, Al-Muwallad MA, et al. (2018). The impact of pharmacists-led medicines reconciliation on healthcare outcomes in secondary care: A systematic review and meta-analysis of randomized controlled trials. PLoS One 13(3): e0193510. DOI: 10.1371/ journal.pone.0193510.

Chen Y, Zhu LL, Zhou Q (2014). Effects of drug pharmacokinetic/ pharmacodynamic properties, characteristics of medication use, and relevant pharmacological interventions on fall risk in elderly patients. Ther Clin Risk Manag 10: 437-448. DOI: 10.2147/TCRM. S63756.

de Jong MR, Van der Elst M, Hartholt KA (2013). Drug-related falls in older patients: implicated drugs, consequences, and possible prevention strategies. Ther Adv Drug Saf 4(4): 147-154. DOI: $10.1177 / 2042098613486829$.

de Vries M, Seppala LJ, Daams JG, van de Glind EMM, Masud T, van der Velde N, EUGMS Task and Finish Group on Fall-RiskIncreasing Drugs (2018). Fall-risk-increasing drugs: a systematic review and meta-analysis: I. cardiovascular drugs. J Am Med Dir Assoc 19(4): 371.e1-371.e9. DOI: 10.1016/j.jamda.2017.12.013.

Deandrea S, Lucenteforte E, Bravi F, Foschi R, La Vecchia C, Negri E (2010). Risk factors for falls in community-dwelling older people: a systematic review and meta-analysis. Epidemiology 21(5): 658-668. DOI: 10.1097/EDE.0b013e3181e89905.

Glab KL, Wooding FG, Tuiskula KA (2014). Medication-related falls in the elderly: mechanisms and prevention strategies. Consult Pharm 29(6): 413-417. DOI: 10.4140/TCP.n.2014.413.

Hajduchová H, Brabcová I, Tóthová V, Bártlová S (2016). Retrospective analysis of falls in selected hospitals of the Czech Republic. Neuro Endocrinol Lett 37(Suppl. 2): 18-24.

Hunderfund ANL, Sweeney CM, Mandrekar JN, Johnson LM, Britton JW (2011). Effect of a multidisciplinary fall risk assessment on falls among neurology inpatients. Mayo Clin Proc 86(1): 19-24. DOI: $10.4065 / \mathrm{mcp} .2010 .0441$.

IBM Micromedex ${ }^{\circledR}$ (2018) - electronic version. Truven health analytics, Greenwood Village, Colorado, USA. [online] [cit. 201807-20]. Available from: http://www.micromedexsolutions.com/

Institute of Health Information and Statistics of the Czech Republic (2018). National database of adverse events. [online] [cit. 201807-15]. Available from: http://shnu.uzis.cz/index.php

Jung D, Shin S, Kim H (2014). A fall prevention guideline for older adults living in long-term care facilities. Int Nurs Rev 61(4): 525-533. DOI: 10.1111/inr.12131.

Krnacova V, Kubena A, Macek K, Bezdek M, Smahelova A, Vlcek J (2012). Severe hypoglycaemia requiring the assistance of emergency medical services - frequency, causes and symptoms. Biomed Pap Med Fac Univ Palacky Olomouc Czech Repub. 156(3): 271-277. DOI: 10.5507/bp.2012.037.

Lord SR, Sherrington C, Menz HB, Close JCT (2011). Falls in older people. Risk factors and strategies for prevention, 2nd ed. Cambridge: University Press.

Lovallo C, Rolandi S, Rossetti AM, Lusignani M (2010). Accidental falls in hospital inpatients: evaluation of sensitivity and specificity of two risk assessment tools. J Adv Nurs 66(3): 690-696. DOI: 10.1111/j.1365-2648.2009.05231.x.

Mackin P (2008). Cardiac side effects of psychiatric drugs. Hum Psychopharmacol 23(Suppl 1): 3-14. DOI: 10.1002/hup.915.

Marvin V, Ward E, Poots AJ, Heard K, Rajagopalan A, Jubraj B (2017). Deprescribing medicines in the acute setting to reduce the risk of falls. Eur J Hosp Pharm 24(1): 10-15. DOI: 10.1136/ ejhpharm-2016-001003.

Miake-Lye IM, Hempel S, Ganz DA, Shekelle PG (2013). Inpatient fall prevention programs as a patient safety strategy: a systematic review. Ann Intern Med 158(5 Pt 2): 390-396. DOI: 10.7326/0003-4819-158-5-201303051-00005.

Milos V, Bondesson Å, Magnusson M, Jakobsson U, Westerlund T, Midlöv P (2014). Fall risk-increasing drugs and falls: a crosssectional study among elderly patients in primary care. BMC Geriatr 14: 40. DOI: 10.1186/1471-2318-14-40.

Mott DA, Martin B, Breslow R, Michaels B, Kirchner J, Mahoney J, Margolis A (2016). Impact of a medication therapy management intervention targeting medications associated with falling: 
Results of a pilot study. J Am Pharm Assoc (2003). 56(1): 22-28. DOI: 10.1016/j.japh.2015.11.001.

Okada K, Okada M, Kamada N, Yamaguchi Y, Kakehashi M, Sasaki H, et al. (2017). Reduction of diuretics and analysis of water and muscle volumes to prevent falls and fall-related fractures in older adults. Geriatr Gerontol Int 17(2): 262-269. DOI: 10.1111/ ggi.12719.

Ribeiro TB, de Melo DO, Maia FDM, Ribeiro E (2018). Medicationrelated inpatient falls: a critical review. Braz J Pharm Sci 54(1): e17355. DOI: 10.1590/s2175-97902018000117355.

Seppala LJ, Wermelink AMAT, de Vries M, Ploegmakers KJ, van de Glind EMM, Daams JG, et al. (2018a). Fall-Risk-Increasing Drugs: A Systematic Review and Meta-analysis: II. Psychotropics. J Am Med Dir Assoc 19(4): 371.e11-371.e17. DOI: 10.1016/j. jamda.2017.12.098.

Seppala LJ, van de Glind EMM, Daams JG, Ploegmakers KJ, de Vries M, Wermelink, AMAT, et al. (2018b). Fall-risk-increasing drugs: a systematic review and meta-analysis: III. Others. J Am Med Dir Assoc 19(4): 372.e1-372.e8. DOI: 10.1016/j. jamda.2017.12.099.
Söderberg KC, Laflamme L, Möller J (2013). Newly initiated opioid treatment and the risk of fall-related injuries. A nationwide, register-based, case-crossover study in Sweden. CNS Drugs 27(2): 155-161. DOI: 10.1007/s40263-013-0038-1.

Walker R, Whittlesea C (2012). Clinical pharmacy and therapeutics. Edinburgh: Churchill Livingstone.

Weddle SC, Rowe AS, Jeter JW, Renwick RC, Chamberlin SM, Franks AS (2017). Assessment of clinical pharmacy interventions to reduce outpatient use of high-risk medications in the elderly. J Manag Care Spec Pharm 23(5): 520-524. DOI: 10.18553/ jmcp.2017.23.5.520.

World Health Organization (2018). Falls. [online] [cit. 2018-07-10]. Available from: http://www.who.int/news-room/fact-sheets/ detail/falls

Zia A, Kamaruzzaman SB, Tan MP (2017). The consumption of two or more fall risk-increasing drugs rather than polypharmacy is associated with falls. Geriatr Gerontol Int 17(3): 463-470. DOI: $10.1111 /$ ggi.12741. 